\title{
Finite Element and Split Bregman Methods for Solving a Family of Optimal Control Problem with Partial Differential Equation Constraint
}

\author{
Mahmoud Lotfi \\ Department of Applied Mathematics, University of Kurdistan, Sanandaj, Iran.
}

\begin{abstract}
In this article we will discuss the solution of elliptic optimal control problem. First, by using the finite element method we obtain the discrete form of the problem. The obtained discrete problem is actually a large scale constrained optimization problem. Solving this optimization problem with traditional methods is difficult and requires a lot of CPU time and memory. But split Bergman method converts the constrained problem to an unconstrained, and hence it saves time and memory requirement. Then we use the split Bregman method for solving this problem, and examples show the speed and accuracy of split Bregman methods for solving this type of problems. We also use the SQP method for solving the examples and compare with split Bregman method.
\end{abstract}

Keywords: Split Bregman Method, Optimal Control with Elliptic Partial Differential Equation Constraint, Finite Element Method.

\section{INTRODUCTION}

In recent decades optimal control problems with partial differential equation constraints, have been studied extensively. These issues are very complex and the numerical solution of such problems is of great importance. In this paper we will discuss the 
numerical solution of the following convex optimal control problem

$$
\begin{aligned}
& \min _{u \in K}\{g(y)+h(u)\}, \\
& -\operatorname{div}(A \nabla y)=f+B u, \quad \text { in } \Omega, \\
& \left.y\right|_{\partial \Omega}=0,
\end{aligned}
$$

where $g$ and $h$ are given convex functionals, $K$ is a closed convex set, and $B$ is a continuous linear operator. Eq. (1) appears, for example, in temperate control problems [12]. Finite element approximation of optimal control problems plays a very important role in numerical methods for these problems. There have been extensively studies on this aspect, see, for example, [24, 3, 9, 4, 6, 21, 13]. Authors in [7] study a finite element method overcoming corner singularities for elliptic optimal control problem posed on a polygon. Gong and Yan in [25] consider the convergence analysis of adaptive finite element method for elliptic optimal control problems with pointwise control constraints. In [29], authors focus on the convergence and quasi-optimality of an adaptive finite element method for elliptic Robin boundary control problems. Hou et al., investigate error estimates and superconvergence of a mixed finite element method for elliptic optimal control problems [22]. According to the accuracy of spectral methods and using the idea of finite element method, Chen et al., in [28], used the spectral element method to solve the Eq (1). They provide the boundaries for the priori and a posteriori error of the method. Here we use the finite element method for discretization of the Eq. (1). The discrete form is obtained as follows

$$
\begin{array}{ll}
\min & g(Y)+h(U), \\
\text { s.t } & A Y=B U+F,
\end{array}
$$

where $A, B \in \mathbb{R}^{n \times n}$ and $F \in \mathbb{R}^{n \times 1}$ is a known matrices. In the finite element method, the accuracy of the solution increases as the number of nodal points get bigger. So in order to obtain sufficient accuracy, it is necessary to select a large number of nodal points as possible. This makes the issue of the discrete form to be relatively large scale. To solve the Eq. (2), there are many optimization methods [28, 18, 17]. In this paper, we use the split Bergman method presented by Goldstein and Osher in [23] for solving Eq. (2).

Solving the optimization problem (2) with traditional methods is difficult and requires a lot of CPU time and memory and accuracy is less. Bregman method is an iterative method that can be used to solve the constrained optimization problems [19]. The characteristic of the Bregman iterative method is speeding in solving problems with high dimensions. It should be noted that the problems derived from finite element method discretization is large, so we use Bregman method to solve the optimization 
problem. The objective function of optimal control problem with elliptic partial differential equation constraint, as the sum of two convex functions, so we use the splite Bregman method, [23] that is useful for this kind of problems. The splite Bregman method is faster than conventional methods, less memory is required and is more accurate. The following numerical example will clearly show these properties. Actually splite Bregman method converts the constrained optimization problem to an unconstrained one. solving the unconstrained problem is much easier than the constrained one.

This paper is organized as follows: In section 2, we describe the finite element approximation for the convex optimal control problem (1) and give the theorems of a priori and a posteriori errors. In section 3, we explain the Bregman iterative method, constrained optimization via Bregman iteration and splite Bregman method. In section 4, we solve the optimization part of problem (1) using the iterative splite Bregman method and the convergence theorem is presented. Numerical results and discussion is presented in section 6 .

\section{THE ELLIPTIC OPTIMAL CONTROL PROBLEM AND FINITE ELEMENT APPROXIMATION}

In this section, we describe the elliptic optimal control problem and its finite element approximation. Let $\Omega$ and $\Omega_{U}$ be two bounded open sets in $\mathbb{R}^{n}(n \leq 3)$ with the Lipschitz boundaries $\partial \Omega$ and $\partial \Omega_{U}$. We denote by $C^{0}(\bar{\Omega})$ the space of continuous functions on $\bar{\Omega}$. We set $H_{0}^{1}=\left\{v \in H^{1}(\Omega):\left.v\right|_{\partial \Omega}=0\right\}$. Suppose that the state space is $\mathbb{Y}=H_{0}^{1}(\Omega)$ and the control space is $\mathbb{U}=L^{2}\left(\Omega_{U}\right)$. In this case, using the inner product $(., .)_{\mathbb{U}}$, with the inner product $(., .)_{U}$, and $H=L^{2}(\Omega)$ with the inner product $(.,$.$) . the weak form of the Eq. (1) will be as follows.$

$$
\begin{array}{ll}
\min & \{g(y)+h(u)\}, \\
\text { s.t } & a(y, w)=(f+B u, w), \quad \forall w \in Y=H_{0}^{1}(\Omega),
\end{array}
$$

where

$$
a(y, w)=\int_{\Omega}(A \nabla v) \cdot \nabla w, \quad \forall v, w \in H^{1}(\Omega)
$$

and

$$
(f, w)=\int_{\Omega} f w, \quad \forall f, w \in L^{2}(\Omega) .
$$

According to these assumptions, the control problem (3) has a unique solution $(y, u)$, and $(y, u)$ is the solution of (3), if and only if there exist a costate variable $p \in \mathbb{Y}$, such 
that $(y, u, p)$ applies in the following conditions [10]:

$$
\begin{array}{ll}
a(y, w)=(f+B u, w), & \forall w \in Y=H_{0}^{1}(\Omega), \\
a(p, q)=\left(g^{\prime}(y), q\right), & \forall q \in Y=H_{0}^{1}(\Omega), \\
\left(h^{\prime}(u)+B^{*} p, v-u\right)_{U} \geq 0, & \forall v \in K=L^{2}\left(\Omega_{U}\right) .
\end{array}
$$

where $B^{*}$ is the adjoint operator of $B$ and $g^{\prime}$ and $h^{\prime}$ are the derivatives of $g$ and $h$ respectively. For simplicity, we consider only $n$-simplex and assume that $\Omega$ and $\partial \Omega$ are polygonal. Let $\mathbf{T}^{h}$ be a partitioning of $\Omega$ into disjoint open regular $n$-simplices $\tau$, so that $\bar{\Omega}=\bigcup_{\tau \in \mathbf{T}^{h}} \bar{\tau}$. Each element has a maximum one face on $\partial \Omega$, and if $\tau$ and $\tau^{\prime} \in \mathbf{T}^{h}$, the adjoining elements $\bar{\tau}$ and $\bar{\tau}^{\prime}$ have either only one common vertex or a whole edge or a whole face. Let $h_{\tau}$ denote the diameter of the element $\tau$ in $\mathbf{T}^{h}$. Associated with $\mathbf{T}^{h}$ is a finite dimensional subspace $S^{h}$ of $C^{0}(\bar{\Omega})$ such that $\left.v_{h}\right|_{\tau}$ are polynomials of $k$-order $(k \geq 1)$ for all $v_{h} \in S^{h}$ and $\tau \in \mathbf{T}^{h}$. Denote $\left\{P_{i}\right\}(i=1,2, \ldots, J)$ the vertex set associated with $\mathbf{T}^{h}$. Let $Y^{h}=V_{0}^{h}:=S^{h} \cap Y$. Similarly, we have a regular partitioning of $\Omega_{U}$, and we use the following corresponding notation: $\mathbf{T}_{U}^{h}, \tau_{U}, h_{\tau U}$ and $P_{i}^{U}\left(i=1,2, \ldots, J_{U}\right)$. Associated with $\mathbf{T}_{U}^{h}$ is another finite dimensional subspace $W_{U}^{h}$ of $L^{2}\left(\Omega_{U}\right)$ such that $\left.v_{h}\right|_{\tau_{U}}$ are polynomials of $k$-order $(k \geq 0)$ for all $v_{h} \in W_{U}^{h}$ and $\tau_{h} \in \mathbf{T}_{U}^{h}$. Note here that there is no requirement for the continuity or boundary conditions. Let $U^{h}=W_{U}^{h} \subset U=L^{2}\left(\Omega_{U}\right)$. Due to the limited regularity of the optimal control $u$ (at most in $H^{1}\left(\Omega_{U}\right)$ in general), here we will consider only the piecewise constant space for the control approximation, while higher-order finite spaces may be used for the state and costate. Then a possible finite element approximation of (3) is as follows:

$$
\begin{aligned}
& \min _{u_{h} \in K^{h}}\left\{g\left(y_{h}\right)+h\left(u_{h}\right)\right\}, \\
& a\left(y_{h}, u_{h}\right)=\left(f+B u_{h}, w_{h}\right), \quad \forall w_{h} \in Y^{h} .
\end{aligned}
$$

where $K^{h}$ is a closed convex set in $U^{h}$ such that there are $v_{h} \in K^{h}$ converging to an element $v \in K$ in $U$. It follows that the control problem (5) has a unique solution $\left(y_{h}, u_{h}\right)$ and $\left(y_{h}, u_{h}\right) \in Y^{h} \times U^{h}$ is the solution of (5) if and only if there is a costate $p_{h} \in Y^{h}$ such that the $\left(y_{h}, p_{h}, u_{h}\right)$ satisfies the following optimality conditions [10]:

$$
\begin{aligned}
& a\left(y_{h}, w_{h}\right)=\left(f+B u_{h}, w_{h}\right), \quad \forall w_{h} \in Y^{h} \subset Y=H_{0}^{1}(\Omega), \\
& a\left(p_{h}, q_{h}\right)=\left(g^{\prime}\left(y_{h}\right), q_{h}\right), \quad \forall q_{h} \in Y^{h} \subset Y=H_{0}^{1}(\Omega), \\
& \left(h^{\prime}\left(u_{h}\right)+B^{*} p_{h}, v_{h}-u_{h}\right)_{U} \geq 0, \quad \forall v_{h} \in K^{h} \subset U=L^{2}\left(\Omega_{U}\right) .
\end{aligned}
$$


The finite element approximation solution must be solved by using some mathematical programming algorithms such as the conjugate gradient method, the interior point method, and the SQP algorithms. In this article we use the split Bregman method to solve the problem.

\subsection{A priori error estimate}

Theorem 1 ([26]). Suppose that $(y, u, p)$ is the exact solution of (4) and $\left(y_{h}, u_{h}, p_{h}\right)$ is the approximate result of finite element method. Then we have

$$
\left\|u-u_{h}\right\|_{0,2, \Omega}^{2}+\left\|y-y_{h}\right\|_{0,2, \Omega}^{2} \leq C h^{4}\|y\|_{2,2, \Omega}^{2}+C h^{4} \log ^{2} h\|p\|_{2, \infty, \Omega}^{2},
$$

and

$$
\left\|u-u_{h}\right\|_{0,1, \Omega}+\left\|p-p_{h}\right\|_{0, \infty, \Omega} \leq C h^{2}|\log h|+C h^{2} .
$$

\subsection{A posteriori error estimate}

Theorem 2 ([26]). Suppose that $(y, u, p)$ is the exact solution of (4) and $\left(y_{h}, u_{h}, p_{h}\right)$ is the approximate result of finite element method. Then we have

$$
\left\|u-u_{h}\right\|_{0,1, \Omega}^{2}+\left\|y-y_{h}\right\|_{0,2, \Omega}^{2}+\left\|p-p_{h}\right\|_{0, \infty, \Omega}^{2} \leq C \eta_{1}^{2}+C \eta_{2}^{2},
$$

where

$$
\begin{aligned}
& \eta_{1}=\left(\sum_{\tau \in \mathbf{T}^{h}} h_{\tau}^{4} \int_{\tau}\left(f+u_{h}+\operatorname{div}\left(A \nabla y_{h}\right)\right)^{2}+\sum_{e \subset \partial \mathbf{T}^{h}} h_{e}^{3} \int_{e}\left[\left(A \nabla y_{h} \cdot n\right)\right]^{2}\right)^{\frac{1}{2}}, \\
& \eta_{2}=\left(\sum_{\tau \in \mathbf{T}^{h}} h_{\tau}^{s}\left\|y_{h}-y_{0}+\operatorname{div}\left(A^{*} \nabla p_{h}\right)\right\|_{0, s, \tau}^{s}+\sum_{e \subset \partial \mathbf{T}^{h}} h_{e}\left\|\left[\left(A^{*} \nabla p_{h} \cdot n\right)\right]\right\|_{0, s, e}^{s}\right)^{\frac{1}{s}}, 1<s<\infty .
\end{aligned}
$$

\section{BREGMAN ITERATIVE METHOD}

Bergman method is a iterative optimization method that first time introduced by Bergman in 1967. He used this method for finding extreme convex functions [14]. Osher et. al. [19] used this method to solve som problems in image processing. Bergman iterative method used for solving the problem of basis pursuit

$$
\begin{aligned}
& \min _{u}\|u\|_{1} \\
& \text { s.t. } A u=f
\end{aligned}
$$


widely [20, 8, 27]. The application of this method in solving medical images has been investigated in [15]. This method can be used to solve constrained optimization problems [19]. Bregman iterative method is defined based on Bregman distance.

definition 1. [14] Suppose $g: \chi \rightarrow \mathbb{R}$ is a convex function and $u \in \chi$. An element $x \in \chi^{*}$ is called a subgradient of $g$ at $u$ if for all $v \in \chi$

$$
g(v)-g(u)-\langle x, u-v\rangle \geq 0
$$

The set of all subgradients of $g$ at $u$ is called the subdifferential of $g$ at $u$, and is denoted by $\partial g(u)$.

definition 2. [14] Suppose $g: \chi \rightarrow \mathbb{R}$ is a convex function, $u, v \in \chi$ and $x \in \partial g(v)$. Then the Bregman distance between points $u$ and $v$ is defined by

$$
D_{g}^{x}(u, v)=g(u)-g(v)-\langle x, u-v\rangle .
$$

The Bregman distance satisfies the following conditions [14]:

1. If $u, v \in \chi$ and $x \in \partial g(v)$, then $D_{g}^{x}(u, v) \geq 0$.

2. $D_{g}^{x}(v, v)=0$.

3. If $w$ between $u$ and $v$, then $D_{g}^{x}(u, v) \geq D_{g}^{x}(w, v)$.

Now suppose there are two convex functions $f$ and $g$ on $\mathbb{R}^{n}$ that are $\min _{u \in \mathbb{R}^{n}} f(u)=0$. Consider the unconstrained optimization problem below

$$
\min _{u \in \mathbb{R}^{n}} g(u)+\alpha f(u) .
$$

Eq. (9) can be solved using Bergman iterative [14]

$$
\begin{aligned}
u^{k+1} & =\min D_{g}^{x}\left(u, u^{k}\right)-\alpha f(u) \\
& =\min g(u)-\left\langle x^{k}, u-u^{k}\right\rangle+\alpha f(u) .
\end{aligned}
$$

In [19], the authors analyze the convergence of Bregman iterative scheme.

Theorem 3. [19] If $f$ and $g$ are convex functions, $f$ is differentiable and Eq. (10) has a solutions, then

$$
\begin{gathered}
f\left(u^{k+1}\right) \leq f\left(u^{k}\right) \\
f\left(u^{k}\right) \leq f\left(u^{*}\right)+\frac{g\left(u^{*}\right)}{k}
\end{gathered}
$$


Now we want to solve constrained optimization problem

$$
\begin{aligned}
& \min _{u} g(u) \\
& \text { s.t. } \quad A u=b
\end{aligned}
$$

where $A$ is a linear operator and $b$ is a vector. Problem (11) can be changed to an unconstrained problem

$$
\min _{u} g(u)+\frac{\alpha}{2}\|A u-b\|_{2}^{2}
$$

which is a special case of problem (9). The Bregman iteration (10) for problem (12) is

$$
\begin{aligned}
u^{k+1} & =\min _{u} D_{g}^{x}\left(u, u^{k}\right)+\frac{\alpha}{2}\|A u-b\|_{2}^{2} \\
= & \min _{u} g(u)-\left\langle x^{k}, u-u^{k}\right\rangle+\frac{\alpha}{2}\|A u-b\|_{2}^{2}, \\
x^{k+1} & =x^{k}-\alpha A^{T}\left(A u^{k+1}-b\right) .
\end{aligned}
$$

If $A$ is linear, the complicated formula (13) can be written down more simply [19]

$$
\begin{aligned}
& u^{k+1}=\min _{u} g(u)+\frac{\alpha}{2}\left\|A u-b^{k}\right\|_{2}^{2}, \\
& b^{k+1}=b^{k}+b-A u^{k} .
\end{aligned}
$$

Equivalence Eq. (13) and Eq. (18) and theorem 3, give the following result

$$
\lim _{k \rightarrow \infty} A u^{k}=b .
$$

The following theorem shows that a solution (18) is a solution to the original constrained problem (11).

Theorem 4. [23] Let $g: \mathbb{R}^{n} \rightarrow \mathbb{R}$ be convex and $A: \mathbb{R}^{n} \rightarrow \mathbb{R}^{m}$ be linear. Consider the algorithm (18). Suppose that some iterate, $u^{*}$, satisfies $A u^{*}=b$. Then $u^{*}$ is a solution to the original constrained problem (11).

\subsection{Split Bregman Method}

Goldstein and Osher used the SBM for solving the below problem [23]

$$
\min _{u}|\phi(u)|+g(u),
$$

where $|$.$| denotes the 11-norm, and both |\phi(u)|$ and $g(u)$ are convex functions. They consider the following problem

$$
\min _{u, d}|d|+g(u)
$$




$$
\text { s.t. } \quad d=\phi(u) \text {. }
$$

To solve this problem, first we convert it into an unconstrained problem

$$
\min _{u, d}|d|+g(u)+\frac{\alpha}{2}\|d-\phi(u)\|_{2}^{2} .
$$

If we let $\Theta(u, d)=|d|+g(u)$, and define $\Psi(u, d)=d-\phi(u)$, then Bergman iteration will be as follows

$$
\begin{aligned}
& \left(u^{k+1}, d^{k+1}\right)=\min _{u, d} D_{\Theta}^{x}\left(u, u^{k}, d, d^{k}\right)+\frac{\alpha}{2}\|d-\phi(u)\|_{2}^{2} \\
& =\min _{u, d} \Theta(u, d)-\left\langle x_{u}^{k}, u-u^{k}\right\rangle-\left\langle x_{d}^{k}, u-u^{k}\right\rangle+\frac{\alpha}{2}\|d-\phi(u)\|_{2}^{2}, \\
& x_{u}^{k+1}=x_{u}^{k}-\alpha(\nabla \phi)^{T}\left(\phi\left(u^{k+1}\right)-d^{k+1}\right), \\
& x_{d}^{k+1}=x_{d}^{k}-\alpha\left(d^{k+1}-\phi\left(u^{k+1}\right)\right) .
\end{aligned}
$$

When we apply the simplification presented in (13), we have

$$
S B M\left\{\begin{array}{l}
\left(u^{k+1}, d^{k+1}\right)=\min _{u, d}|d|+g(u)+\frac{\alpha}{2}\left\|d-\phi(u)-b^{k}\right\|_{2}^{2}, \\
b^{k+1}=b^{k}+\left(\phi\left(u^{k+1}\right)-d^{k+1}\right) .
\end{array}\right.
$$

In order to implement the algorithm (17), we must be able to solve the problem

$$
\left(u^{k+1}, d^{k+1}\right)=\min _{u, d}|d|+g(u)+\frac{\alpha}{2}\left\|d-\phi(u)-b^{k}\right\|_{2}^{2} .
$$

As a result, we need to do the following three steps

$$
\begin{aligned}
& \text { Step } 1: u^{k+1}=\min _{u} g(u)+\frac{\alpha}{2}\left\|d^{k}-\phi(u)-b^{k}\right\|_{2}^{2}, \\
& \text { Step2: } d^{k+1}=\min _{d}|d|+\frac{\alpha}{2}\left\|d-\phi\left(u^{k+1}\right)-b^{k}\right\|_{2}^{2}, \\
& \text { Step3: } b^{k+1}=b^{k}+\left(\phi\left(u^{k+1}\right)-d^{k+1}\right) .
\end{aligned}
$$

The speed of the SBM is largely dependent on how fast we can solve each of these subproblems [23]. 


\section{SOLVING THE OPTIMIZATION PART OF ELLIPTIC OPTIMAL CONTROL PROBLEM USING THE ITERATIVE SBM}

In this section, we consider the convex optimal control problem:

$$
\begin{aligned}
& \min \frac{1}{2} \int_{\Omega}\left(y-y_{0}\right)^{2}+\frac{1}{2} \int_{\Omega_{U}} u^{2}, \\
& -\operatorname{div}(A \nabla y)=B u+f, \quad \text { in } \Omega, \\
& \left.y\right|_{\partial \Omega}=0 .
\end{aligned}
$$

We assume that, $\Omega_{U}=\Omega=[0,1] \times[0,1]$ and $B=I$. We also use the same meshes for the approximation of the state and the control. Thus $\tau_{U}=\tau$. Let $\Omega^{h}$ be a polygonal approximation to $\Omega$ with boundary $\partial \Omega^{h}$. Let $\mathbf{T}^{h}$ be a partitioning of $\Omega^{h}$ into a disjoint regular triangular $\tau$ so that $\bar{\Omega}^{h}=U_{\tau \in \mathbf{T}^{h}} \bar{\tau}$. Assume that the state $y$ is approximated in the finite element space $Y^{h}$ with $\Phi^{i}$ as basis functions and $u$ is approximated in $U^{h}$ with $\Psi^{i}$ as basis functions. Thus the problem (18) is discretized as the following optimization problem:

$$
\begin{aligned}
& \min \frac{1}{2}\left(Y-Y^{0}\right)^{T} Q\left(Y-Y^{0}\right)+\frac{1}{2} U^{T} Q U, \\
& \text { s.t } \quad A Y=B U+F,
\end{aligned}
$$

with

$$
\begin{array}{ll}
Q^{i j}=\int_{\Omega} \Phi^{i} \Phi^{j} d x, & M^{i j}=\int_{\Omega} \Psi^{i} \Psi^{j} d x, \\
A^{i j}=\int_{\Omega} \nabla \Phi^{i} \nabla \Phi^{j} d x, & B^{i j}=\int_{\Omega} \Phi^{i} \Psi^{j} d x, \\
F_{i}=\int_{\Omega} f \Phi^{j} d x . &
\end{array}
$$

With these assumptions iterative Bergman method to the problem (19) is obtained as follows:

$$
\begin{gathered}
\left(Y^{k+1}, U^{k+1}\right)=\min G(Y)-G\left(Y^{k}\right)-\left\langle p_{Y}^{k}, Y-Y^{k}\right\rangle+M(U) \\
-\left\langle p_{U}^{k}, U-U^{k}\right\rangle+\frac{\alpha}{2}\|B U+F-A U\|_{2}^{2}, \\
p_{Y}^{k+1}=p_{Y}^{k}+\alpha B^{T}\left(B U^{k+1}+F-A Y^{k+1}\right), \\
p_{U}^{k+1}=p_{U}^{k}+\alpha A^{T}\left(B U^{k+1}+F-A Y^{k+1}\right),
\end{gathered}
$$

where $G(Y)=\frac{1}{2}\left(Y^{h}-Y^{0}\right)^{T} Q\left(Y^{h}-Y^{0}\right)$ and $N(U)=\frac{1}{2} U^{h^{T}} Q U^{h}$. 
But according to the properties of the problem, split Bregman method is more suitable:

Split Bregman method iterative

$$
\begin{aligned}
& Y^{k+1}=\min G(Y)+\left\langle\lambda^{k},-A Y\right\rangle+\frac{\alpha}{2}\left\|B U^{k}+F-A Y\right\|_{2}^{2}, \\
& U^{k+1}=\min M(U)+\left\langle\lambda^{k}, B U\right\rangle+\frac{\alpha}{2}\left\|B U+F-A Y^{k+1}\right\|_{2}^{2}, \\
& \lambda^{k+1}=\lambda^{k}+\alpha\left(B U^{k+1}+F-A Y^{k+1}\right) .
\end{aligned}
$$

The speed of the Bregman splitting method is largely dependent on how fast we can solve subproblems. To solve these subproblems can be direct method and methods of used to solve linear systems.

\section{CONVERGENCE THEOREM}

Associated to the primal problem (19) is the Lagrangian:

$$
L(Y, U, \lambda)=G(Y)+M(U)+\langle\lambda, B U+F-A Y\rangle,
$$

where the dual variable $\lambda \in \mathbb{R}^{d}$ can be thought of as a vector of Lagrange multipliers. The dual functional $q(\lambda)$ is a concave function $q: R^{d} \rightarrow[-\infty, \infty)$ defined by:

$$
q(\lambda)=\inf L(Y, U, \lambda)
$$

The dual problem to (19) is:

$$
\max q(\lambda)
$$

Finding optimal solutions of (19) and (20) is equivalent to finding a saddle point of $L$. More precisely, $\left(Y^{*}, U^{*}\right)$ is an optimal primal solution and $\lambda^{*}$ is an optimal dual solution if and only if:

$$
L\left(Y^{*}, U^{*}, \lambda\right) \leq L\left(Y^{*}, U^{*}, \lambda^{*}\right) \leq L\left(Y, U, \lambda^{*}\right), \quad \forall Y, U, \lambda .
$$

Theorem 5. [5] Let $\lambda^{0}$ and $Y^{0}$ be arbitrary and let $\alpha>0$. Suppose we are also given sequences $\left\{\mu_{k}\right\}$ and $\left\{\nu_{k}\right\}$ such that :

$$
\mu_{k} \geq 0, \nu_{k} \geq 0, \sum_{k=0}^{\infty} \mu_{k}<\infty, \sum_{k=0}^{\infty} \nu_{k}<\infty
$$

Suppose that:

$$
\left\|Y_{k+1}^{h}-\min G\left(Y^{h}\right)+\left\langle\lambda^{k},-A Y^{h}\right\rangle\right\|+\frac{\alpha}{2}\left\|B U_{k}^{h}+F-A Y^{h}\right\|_{2}^{2} \leq \mu_{k},
$$


and

$$
\left\|U_{k+1}^{h}-\min N\left(Y^{h}\right)+\left\langle\lambda^{k}, B U^{h}\right\rangle\right\|+\frac{\alpha}{2}\left\|B U^{h}+F-A Y_{k}^{h}\right\|_{2}^{2} \leq \nu_{k} .
$$

If there exists a saddle point of $L(Y, U, \lambda)$, then

$$
\lambda^{k} \rightarrow \lambda^{*}, \quad U^{k} \rightarrow U^{*}, \quad Y^{k} \rightarrow Y^{*}
$$

where $\left(Y^{*}, U^{*}, \lambda^{*}\right)$ is such a saddle point. On the other hand, if no such saddle point exists, then at least one of the sequences $\left\{\mu_{k}\right\}$ or $\left\{\nu_{k}\right\}$ must be unbounded.

\section{NUMERICAL EXAMPLES}

In this section, we carry out some numerical examples to demonstrate our theoretical results. the state and control variables were approximated by piecewise linear functions. In our examples, we choose the domain $\Omega_{U}=\Omega=[0,1] \times[0,1]$ and $B=I$. Our examples was solved using MATLAB softwar. In split Bregman method put $\alpha=1$. Stop condition for split Bregman method is considered as follows:

$$
\frac{\|B U+F-A Y\|}{\|F\|} \leq 10^{-6} .
$$

We also use the SQP method for solving the problem and compare its results with these of the split Bregman method.

Example 1. In this example we solve the following elliptic control problem:

$$
\begin{aligned}
& \min \frac{1}{2} \int_{\Omega}\left(y-y_{0}\right)^{2} d x+\frac{1}{2} \int_{\Omega} u^{2} d x, \\
& \text { s.t } \quad-\Delta y=B u+f, \quad y\left\|_{\partial \Omega}=0 .\right\|,
\end{aligned}
$$

where

$$
\begin{array}{ll}
y=\frac{1}{4} \sin \left(2 \pi x_{1}\right) \sin \left(2 \pi x_{2}\right), & y_{0}=y-16 \pi^{2} y, \\
u=-2 y, & f=8 \pi^{2} y+2 y .
\end{array}
$$

\section{Solve by finite element method with uniform mesh}

Table 1 showe the $L_{2}$-error for $y_{h}$ and $u_{h}$ with different value of elements. Results clearly show the efficiency of SBM compared to SQP method. Figures 1 and 2, show graphs of approximate solution of $u$ and $y$ and absolute error $\left|y-y_{h}\right|$ and $\left|u-u_{h}\right|$ with 1089 element. Figures 3, show graphs of error of split Bregman iteration. 
Table 1: Numerical results for example 1 with different value of elements on uniform mesh.

\begin{tabular}{cc|rrr|rrr}
\hline \hline & & \multicolumn{3}{|c|}{ With SBM } & \multicolumn{3}{c}{ With SQP } \\
Nodes & Elements & $\left\|y-y_{h}\right\|_{2}$ & $\left\|u-u_{h}\right\|_{2}$ & Times (s) & $\left\|y-y_{h}\right\|_{2}$ & $\left\|u-u_{h}\right\|_{2}$ & Times (s) \\
\hline 289 & 512 & 0.0193 & 0.0032 & 0.024480 & 0.0193 & 0.0032 & 12.560520 \\
1089 & 2048 & 0.0050 & $7.99063 \mathrm{E}-4$ & 0.0326862 & 0.0058 & $7.8488 \mathrm{E}-4$ & 243.469522 \\
4225 & 8192 & 0.0013 & $1.9743 \mathrm{E}-4$ & 4.259551 & - & - & - \\
16641 & 32768 & $3.148 \mathrm{E}-4$ & $4.9335 \mathrm{E}-5$ & 72.658772 & - & - & - \\
\hline
\end{tabular}
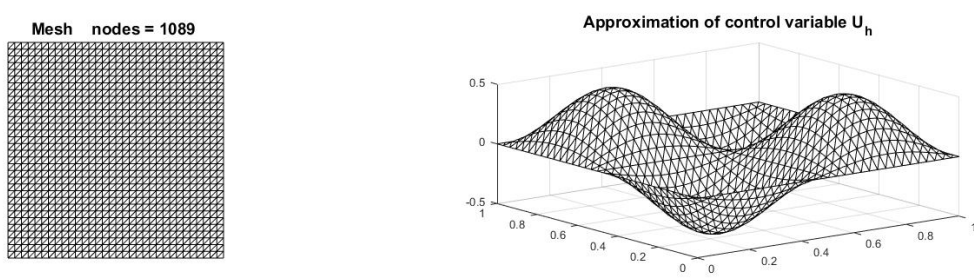

Exact solution U

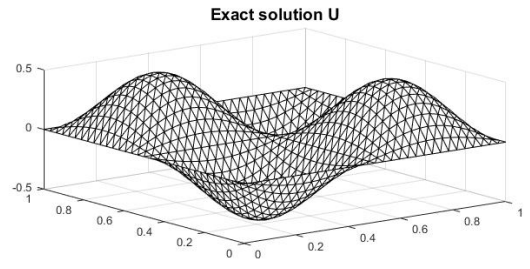

$\left|\mathrm{U}-\mathrm{U}_{\mathrm{h}}\right|$

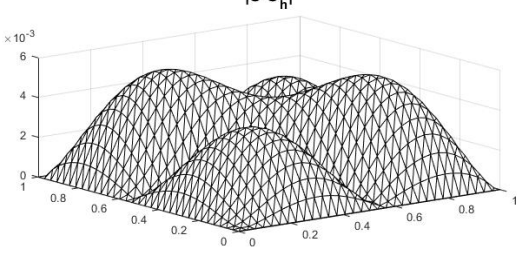

Fig. 1: Graph the results of finite element and SBM for Example 1 (state variable).

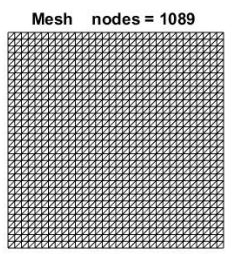

Exact solution $u$

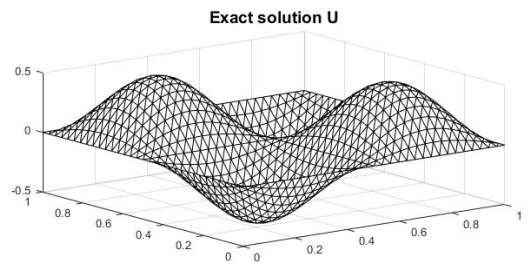

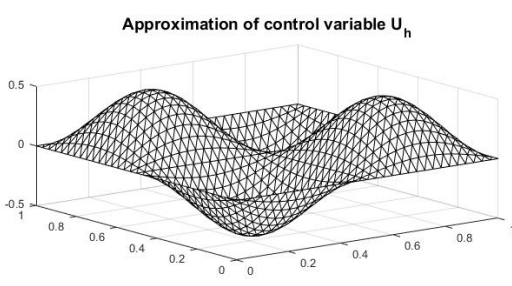

$\left|\mathrm{U}-\mathrm{U}_{\mathrm{h}}\right|$

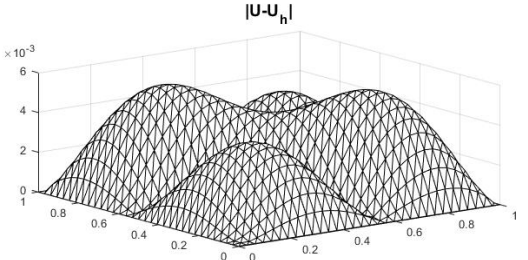

Fig. 2: Graph the results of finite element and SBM for Example 1 (control variable). 


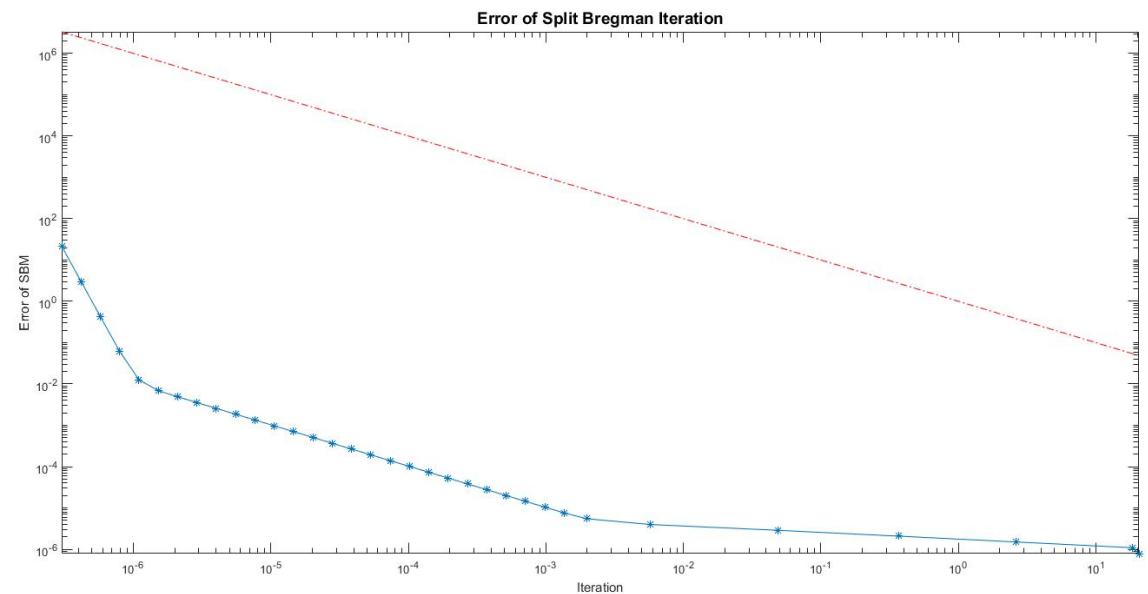

Fig. 3: Error of split Bregman iteration for Example 1.

\section{Solve by adaptive finite element method}

In Table 2, the results of adaptive finite element method are presented with both methods SBM and SQP method. The results of this table clearly show the difference between adaptive and uniform mesh. Figure 4 show the results of adaptive finite element method for both variables $u_{h}$ and $y_{h}$ and adaptive mesh.

Table 2: Numerical results for example 1 with adaptive finite element method.

\begin{tabular}{cc|rrr|rrr}
\hline \hline & & \multicolumn{3}{|c|}{ With SBM } & \multicolumn{3}{c}{ With SQP } \\
Nodes & Elements & $\left\|y-y_{h}\right\|_{2}$ & $\left\|u-u_{h}\right\|_{2}$ & Times (s) & $\left\|y-y_{h}\right\|_{2}$ & $\left\|u-u_{h}\right\|_{2}$ & Times (s) \\
\hline 551 & 1036 & $7.2984 \mathrm{E}-5$ & $7.4025 \mathrm{E}-4$ & 9.672852 & $7.2434 \mathrm{E}-4$ & 0.0071 & 721.909673 \\
\hline
\end{tabular}

Example 2. We consider the following elliptic optimal control problem:

$$
\begin{aligned}
& \min \frac{1}{2} \int_{\Omega}\left(y-y_{0}\right)^{2} d x+\frac{1}{2} \int_{\Omega} u^{2} d x, \\
& \text { s.t } \quad-\Delta y=B u+f,\left.\quad y\right|_{\partial \Omega}=0 .
\end{aligned}
$$

where

$$
\begin{aligned}
& f=4 \pi^{2} \sin \left(2 \pi x_{1}\right)\left(2 \cos \left(2 \pi x_{2}\right)-1\right)+\sin \left(\pi x_{1}\right) x_{2}\left(x_{2}-1\right), \\
& \left.y_{0}=\sin \left(\pi x_{1}\right)\left(2-\pi^{2} x_{2}\left(x_{2}-1\right)\right)-1\right)+\sin \left(2 \pi x_{1}\right)\left(\cos \left(2 \pi x_{2}\right)-1\right), \\
& y=\sin \left(2 \pi x_{1}\right)\left(\cos \left(2 \pi x_{2}\right)-1\right), \\
& u=-\sin \left(\pi x_{1}\right) x_{2}\left(x_{2}-1\right) .
\end{aligned}
$$




\section{Solve by finite element method with uniform mesh}

The results of this example can be seen in table 3. It can be seen from Table 3 that the SBM results are in good agreement with SQP method in CPU time and accuracy. Figures 5 and 6, show graphs of approximate solution of $u$ and $y$ and absolute error $\left|y-y_{h}\right|$ and $\left|u-u_{h}\right|$ with 1089 element. Figures 7, show graphs of error of split Bregman iteration.

Table 3: Numerical results for example 2 with different value of elements on uniform mesh.

\begin{tabular}{cc|rrr|rrr}
\hline \hline & & \multicolumn{3}{|c|}{ With SBM } & \multicolumn{3}{c}{ With SQP } \\
Nodes & Elements & $\left\|y-y_{h}\right\|_{2}$ & $\left\|u-u_{h}\right\|_{2}$ & Times (s) & $\left\|y-y_{h}\right\|_{2}$ & $\left\|u-u_{h}\right\|_{2}$ & Times (s) \\
\hline 289 & 512 & 0.0027 & 0.0140 & 0.0225456 & 0.0027 & 0.0140 & 2.624531 \\
1089 & 2048 & $6.7681 \mathrm{E}-4$ & 0.0035 & 0.355750 & $7.6762 \mathrm{E}-4$ & 0.0035 & 44.563634 \\
4225 & 8192 & $1.6995 \mathrm{E}-4$ & $8.7794-4$ & 6.146724 & - & - & - \\
16641 & 32768 & $4.2635 \mathrm{E}-4$ & $2.1949 \mathrm{E}-5$ & 123.521323 & - & - & - \\
\hline
\end{tabular}

\section{Solve by adaptive finite element method}

In Table 4, the results of adaptive finite element method are presented with both methods SBM and SQP method. The results of this table clearly show the difference between adaptive and uniform mesh. Figure 8 show the results of adaptive finite element method for both variabes $u_{h}$ and $y_{h}$ and adaptive mesh.

Table 4: Numerical results for example 1 with adaptive finite element method.

\begin{tabular}{cc|rcr|rrr}
\hline \hline & & \multicolumn{3}{|c|}{ With SBM } & \multicolumn{3}{c}{ With SQP } \\
Nodes & Elements & $\left\|y-y_{h}\right\|_{2}$ & $\left\|u-u_{h}\right\|_{2}$ & Times (s) & $\left\|y-y_{h}\right\|_{2}$ & $\left\|u-u_{h}\right\|_{2}$ & Times (s) \\
\hline 576 & 1103 & 0.0068 & & 10.987756 & 0.0068 & & 1405.517984 \\
805 & 1538 & & $1.5919 \mathrm{E}-5$ & 14.759973 & & $9.2128 \mathrm{E}-4$ & 3241.436792 \\
\hline
\end{tabular}




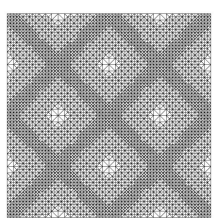

(a) Adaptive mesh

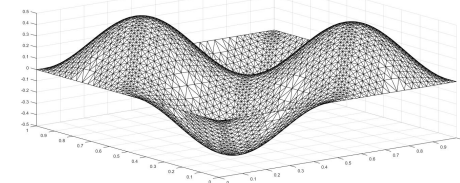

(b) $y_{h}$

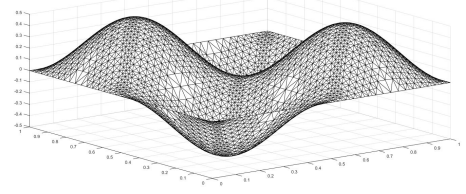

(c) $u_{h}$

Fig. 4: Adaptive finite element method for both variables $u_{h}$ and $y_{h}$ and adaptive mesh for example 1.
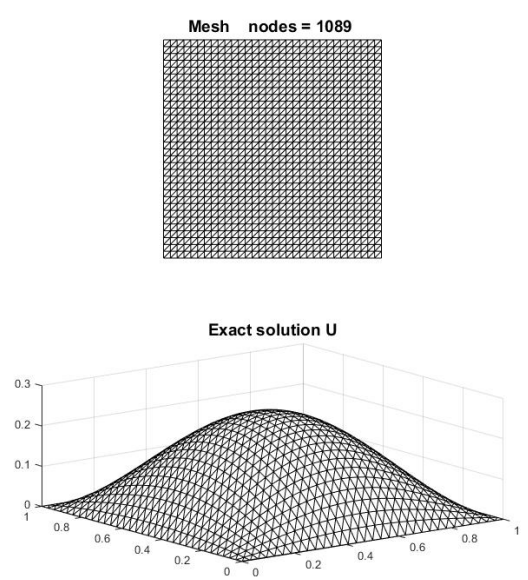

Approximation of control variable $U_{h}$

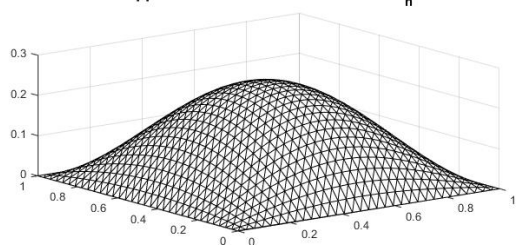

Error U $L^{2}$ error $=6.768370 \mathrm{e}-04$

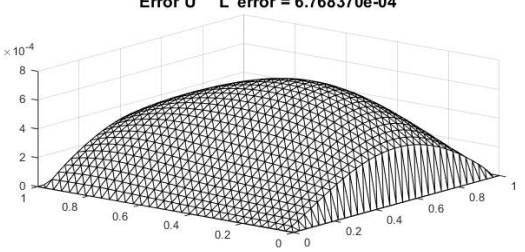

Fig. 5: Graph the results of finite element and SBM for Example 2 (state variable). 

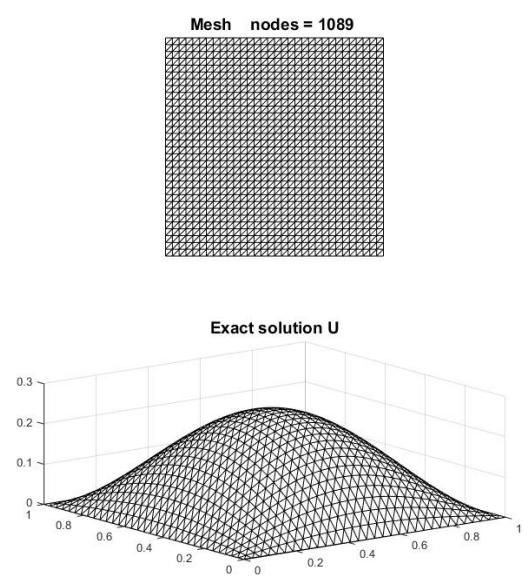

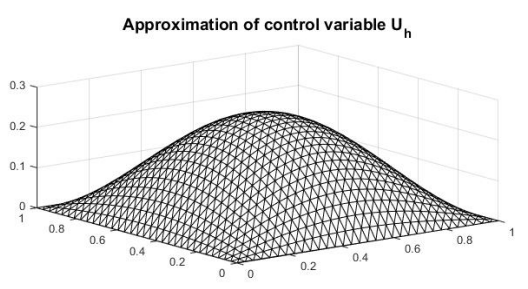

Error U $\quad L^{2}$ error $=6.768370 \mathrm{e}-04$

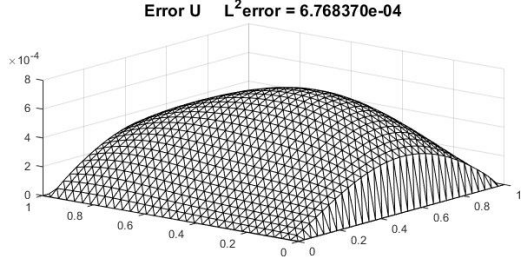

Fig. 6: Graph the results of finite element and SBM for Example 2 (control variable).

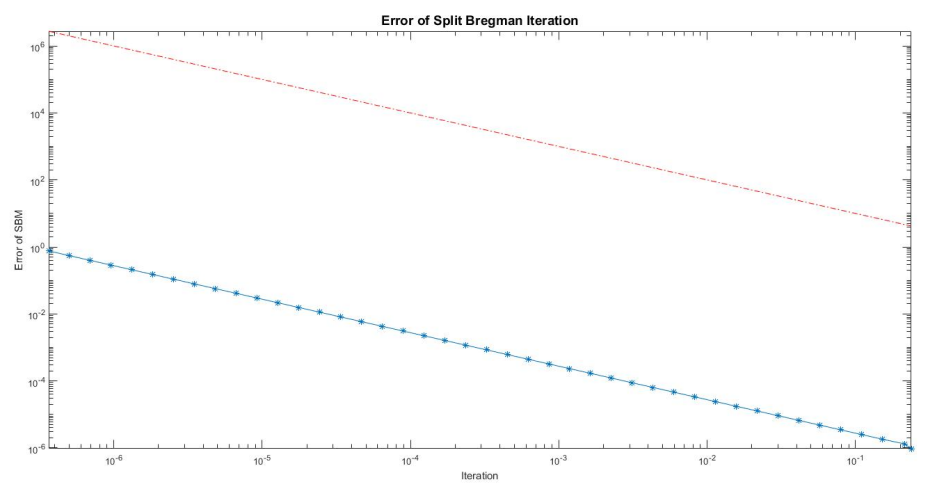

Fig. 7: Error of split Bregman iteration for Example 2.

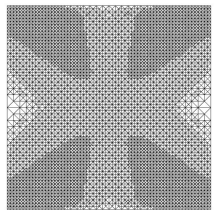

(a) Adaptive mesh

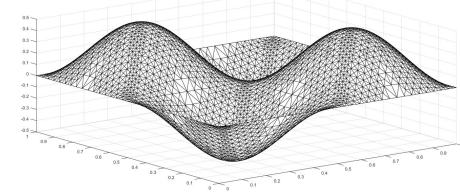

(b) $y_{h}$

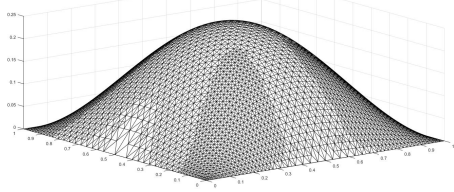

(c) $u_{h}$

Fig. 8: Adaptive finite element method for both variables $u_{h}$ and $y_{h}$ and adaptive mesh for example 1. 


\section{CONCLUSIONS}

The numerical results obtained for the above examples clearly shows the speed and accuracy of split Bregman method in solving this kind of problems. Actually the split Bregman method by conversion of large scale constrained optimization problem to the unconstrained optimization problem of same scale, coused we can use the unconstrained optimization techniques. In this paper, the conditions of problem allows us to solve the unconstrained subproblems directly and derivative with respect to the variable. In example 1 with 1089 nodal points, the time it SQP method for run the program needs (243.469522 second) almost 7448 times the amount of time that split Bergman method (0.0326862 second) to achieve the approximate solution of same accuracy is required. The amount in example 2 is approximately 125. Also, according to numerical results, this accuracy and CPU time can be obtained by applying the adaptive finite element method to a much smaller number of elements.

\section{REFERENCES}

[1] A. Ern, J.L. Guermond, Theory and Practice of Finite Elements, New York, NY: Springer-Verlag 2004.

[2] C. Pozrikidis, Introduction to Finite and Spectral Element Methods Using Matlab, Chapman and Hall/CRC 2005.

[3] D.A. French, J.T. King, Approximation of an elliptic control problem by the finite element method, Numer. Funct. Anal. Optim., (1991) 299-315.

[4] D. Tiba, Lectures on the Optimal Control of Elliptic Equations University of Jyvaskyla Press, Jyvaskyla 1995.

[5] E. Esser, Applications of Lagrangian-based alternating direction methods and connections to split Bregman, UCLA CAM Report (2009) 09-31.

[6] F.S. Falk, Approximation of a class of optimal control problems with order of convergence estimates, J. Math. Anal. Appl., (1973) 28-48.

[7] J.C. Hyung, W.1 Choi and Y. Koh, A finite element method for elliptic optimal control problem on a non-convex polygon with corner singularities, Computers and Mathematics with Applications, 75, (2018) 45-58.

[8] J.F. Cai, S. Osher, and Z Shen, Linearized Bregman iterations for compressed sensing, Ucla Cam Report., (2009) 1515-1536. 
[9] J. Haslinger, P. Neittaanmaki, Finite Element Approximation for Optimal Shape Design, Wiley, Chichester 1989.

[10] J.L. Lions, Optimal Control of Systems Governed by Partial Differential Equations, Springer-Verlag, Berlin 1971.

[11] J.S.

Hesthaven, S. Gottlieb and D. Gottlieb, Spectral Method for Time-Dependent Problems, Cambridge University Press 2007.

[12] G. Duvaut, J.L. Lions, The Inequalities in Mechanics and Physics, Springer, Berlin 1973.

[13] K. Malanowski, Convergence of approximations vs. regularity of solutions for convex, control constrained, optimal control systems, Appl. Math. Optim., (1982) 69-95.

[14] L.M. Bregman, The relaxation method of finding the common point of convex sets and its application to the solution of problems in convex programming, USSR Computational Mathematics and Mathematical Physics., (1967) 200-217.

[15] L. He, T. Chiun Chang, and S. Osher, Mr image reconstruction from sparse radial samples by using iterative refinement procedures, Proceedings of the 13th annual meeting of ISMRM, (2006) 06-35.

[16] L.N. Hand, J.D. Finch, Analytical Mechanics, Cambridge University Press 2008.

[17] M. Hinze, Optimal and Instantaneous Control of the Instationary Navier-Stokes Equations, Habilitation thesis, Technische Universitat Berlin, Berlin, Germany 2000.

[18] S.B. Hazra, Large-Scale PDE-Constrained Optimization in Applications, Springer-Verlag Berlin Heidelberg 2010.

[19] S. Osher, M. Burger, D. Goldfarb, J. Xu, and W. Yin, An iterative regularization method for total variation-based image restoration, MMS, (2005) 460-489.

[20] S. Osher, Y. Mao, B. Dong, and W. Yin, Fast linearized Bregman iterations for compressed sensing and sparse denoising, UCLA CAM Report., (2010) 08-37.

[21] T. Geveci, On the approximation of the solution of an optimal control problem governed by an elliptic equation, Rairo Anal. Num'er., (1979) 313-328. 
[22] T. Hou, C. Liu and Y. Yang, Error estimates and superconvergence of a mixed finite element method for elliptic optimal control problems, Computers and Mathematics with Applications, 74, (2017) 714-726.

[23] T. Goldstein, S. Osher, The split Bregman method for L1-regularized problems, Siam J. Imaging Sci., (2009) 323-343.

[24] W. Alt, On the approximation of infinite optimization problems with an application to optimal control problems, Appl. Math. Optim., (1984) 15-27.

[25] W. Gong, N. Yan, Adaptive finite element method for elliptic optimal control problems: convergence and optimality, Numer. Math., 135, (2017) 1121-1170.

[26] W. Gong, N. Yan, Robust error estimates for the finite element approximation of elliptic optimal control problems, Journal of Computational and Applied Mathematics, 236 (2011) 1370-1381.

[27] W. Yin, S. Osher, D. Goldfarb, and J. Darbon, Bregman iterative algorithms for 11-minimization with applications to compressed sensing, Siam J. Imaging Sci., (2008) 142-168.

[28] Y. Chen, N. Yi, and W. Liu, A Legendre-galerkin spectral method for optimal control problemsgoverned by elliptic equations, Siam J. Numer. Anal, 46(5) (2008) 2254-2275.

[29] Y. Shen, N. Yan and Z. Zhou, Convergence and quasi-optimality of an adaptive finite element method for elliptic Robin boundary control problem, Journal of Computational and Applied Mathematics, Volume 356, (2019) 1-21. 